
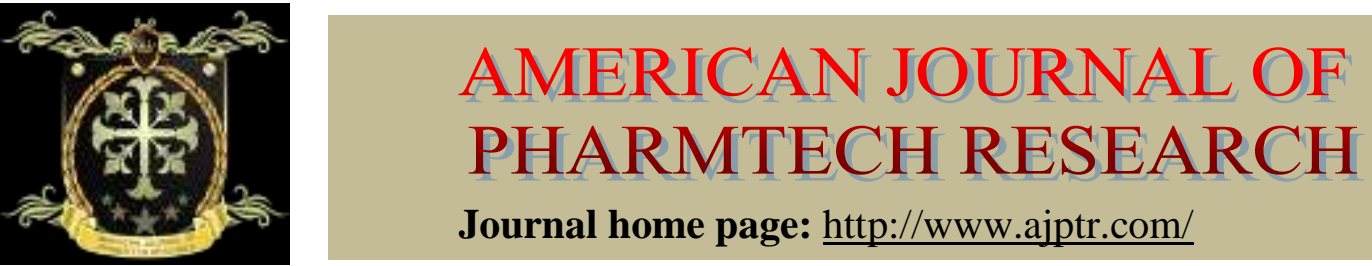

Journal home page: http://www.ajptr.com/

\title{
Design and Evaluation of Controlled Release Gastric Floating Drug Delivery System of Proton Pump Inhibitor
}

Jyothi $\mathbf{M}^{\mathbf{1}}$, Richitha $\mathbf{S}^{\mathbf{1}}$, Sindhu $\mathbf{S}^{\mathbf{1}}$, Hemanth $\mathbf{G}^{\mathbf{1 *}^{*}}$

Department of Pharmaceutics. East Point College Of Pharmacy Bangalore, Karnataka, India

\section{ABSTRACT}

The purpose of this research was to prepare and evaluate a floating drug delivery system of a proton pump inhibitor (pantoprazole sodium). Pantoprazole is a proton pump inhibitor, belongs to group of benzimidazole, used for the treatment of gastric and duodenum ulcers. The core tablets were prepared by direct compression method (effervescent method) by using microcrystalline cellulose, sodium bicarbonate, citric acid, magnesium stearate and HPMC K100 and HPMC K15. The physicochemical parameters like pre-compression and post compression evaluation were performed as per Pharmacopoeial standard and the compatibility study was performed by FTIR and DSC methods. The release data were subjected to different models in order to evaluate their release kinetics and mechanisms. The compatibility study of the prepared Pantoprazole sodium tablets implies the information about no interaction between drug and polymer. The drug release kinetics was observed by Non-Fickian diffusion mechanism. HPMC K100 shows better release properties than HPMC K15. The floating lag time were found to be significantly increased with the increasing concentration of the gas generating agent. After the dissolution study of prepared Pantoprazole sodium floating tablet, it was concluded that the formulations with HPMC K100 shows better controlled release effect than HPMC K15. The release kinetics data implies that the release mechanism of the all formulation was Non-Fickian. The developed floating tablets of pantoprazole sodium may be used in clinic for prolonged drug release for at least $12 \mathrm{~h}$, thereby improving the bioavailability and patient compliance.

Keywords: Pantoprazole sodium, Gastro retentive, Floating drug delivery systems, Release kinetics. Buoyancy studies.

*Corresponding Author Email: hemanthhemu63639@gmail.com

Received 01November 2018, Accepted 20 November 2018

Please cite this article as: Hemanth $\mathrm{G}$ et al., Design and Evaluation of Controlled Release Gastric Floating Drug Delivery System of Proton Pump Inhibitor . American Journal of PharmTech Research 2019. 


\section{INTRODUCTION}

The oral delivery of drugs is by far the most preferable route of drug delivery due to the ease of administration, patient compliance and flexibility in formulation, etc. from immediate release to site-specific delivery, oral dosage forms have really progressed ${ }^{1}$. Controlled drug delivery systems provide drug release at a predetermined, predictable and controlled rate to achieve high therapeutic efficiency with minimal toxicity ${ }^{2}$. Effective oral drug delivery may depend upon the factor such as gastric emptying process, gastrointestinal transit time of dosage form, drug release from the dosage form and site of absorption of drugs $^{3}$. Gastro retentive system can remain in the gastric region for several $\mathrm{h}$ and hence significantly prolong the gastric residence time of drugs. Gastro retention helps to provide better availability of new products with new therapeutic possibilities and substantial benefits for patients ${ }^{4}$. Floating drug delivery system also known as hydro dynamically balanced system, have a bulk density than gastric fluids and thus remain buoyant in the gastric fluid for a prolonged period of time without affecting the gastric emptying rate ${ }^{5}$.Hydro dynamically balanced drug delivery system, in either $\mathrm{t}$ or capsule form, is designed to prolong gastrointestinal (GI) residence time in an area of GI tract, it is prepared by incorporating a high level $(20-70 \% \mathrm{w} / \mathrm{w})$ of one or more gel forming hydrocolloids ${ }^{6}$. The model drug selected for the study was pantoprazole sodium. It is substituted benzimidazole derivative that targets gastric acid proton pumps, the final common pathway for gastric acid secretion. The drug covalently binding to the proton pumps, causing prolonged inhibition of gastric acid secretion. Floating drug delivery systems (FDDS) have bulk density lesser than gastric fluid, so they remain buoyant in the stomach without affecting the gastric emptying rate for a prolonged period of time ${ }^{7}$.Floating drug delivery offers several applications for drugs having poor bioavailability because of the narrow absorption window in the upper part of the gastrointestinal tract. It retains the dosage form at the site of absorption and thus enhances the bioavailability ${ }^{8}$.

\section{MATERIALS AND METHOD}

\section{Materials}

Pantoprazole sodium was collected from Sun pharmaceutical Ltd, Mumbai, India. Polyvinyl pyrrolidone \& Microcrystalline cellulose were obtained from Sd fine chem. Pvt. Ltd., Mumbai, India. HPMC K100 \& HPMC K 15 were taken from Cipla Pvt, Ltd, Bangalore, India. Magnesium stearate \& Talc was obtained from Qualigens fine chemicals, India. Citric acid from Medreich Ltd. \& Sodium bi carbonate from Merck specialties Pvt. Ltd. Mumbai, India.

\section{Methods}




\section{Preformulation studies}

Preformulation testing is the first step in the rationale development of dosage forms of a drug substance. It is performed by solubility, melting point determination \&IR spectroscopy to determine the compatibility of the polymers.

\section{Preparation of standard graph for pantoprazole sodium using pH 6.8 Phosphate Buffer Determination of $\lambda$ max}

$100 \mathrm{mg}$ of pantoprazole sodium was weighed accurately and dissolved in $100 \mathrm{ml}$ of $\mathrm{pH} 6.8$ phosphate buffer in $100 \mathrm{ml}$ volumetric flask (stock solution). $2 \mathrm{ml}$ was taken from the stock solution and transferred into $100 \mathrm{ml}$ volumetric flask and diluted up to $100 \mathrm{ml}$ with pH6.8 phosphate buffer. The resulting solution was labeled as standard working Solution. $2 \mathrm{ml}$ of the working solution was withdrawn and diluted up to $10 \mathrm{ml}$ with $\mathrm{pH} 6.8$ phosphate buffer in $10 \mathrm{ml}$ volumetric flask. The spectrum of this solution was run in 200 to $400 \mathrm{~nm}$ range in UV-visible spectrophotometer. The $\lambda$ max of the pantoprazole sodium was found to be $288.7 \mathrm{~nm}$.

\section{Preparation of standard graph using phosphate buffer pH6.8}

From standard working solution, $1 \mathrm{ml}, 2 \mathrm{ml}, 3 \mathrm{ml}, 4 \mathrm{ml}, 5 \mathrm{ml}$ and $6 \mathrm{ml}$ was withdrawn and diluted up to $10 \mathrm{ml}$ with $\mathrm{pH} 6.8$ phosphate buffer in $10 \mathrm{ml}$ volumetric flask to get concentration of $2 \mu \mathrm{g}, 4 \mu \mathrm{g}, 6 \mu \mathrm{g}, 8 \mu \mathrm{g}, 10 \mu \mathrm{g}$ and $12 \mu \mathrm{g}$ and $12 \mu \mathrm{g}$ respectively. The absorbance of each solution was measured by UV-visible spectrophotometer at $288.7 \mathrm{~nm}$ using the $\mathrm{pH} 6.8$ phosphate buffer as blank.

\section{Preparation of pantoprazole sodium tablets}

Floating tablets containing pantoprazole sodium were prepared by direct compression technique using variable concentration of HPMC K15, HPMC K100, and other ingredients like MCC, sodium bicarbonate, citric acid and PVP.

Different tablet formulations were prepared by direct compression method. All the powders were passed through 60 mesh sieve the required qty. of drug and lower density polymer were mixed geometrically and then tablets are compressed in compression machine at specified pressure with $100 \mathrm{~mm}$ round punch. Refer Table 1.

\section{Pre-compression parameters ${ }^{9}$}

The pre-compression parameters were determined by performing bulk density, tapped density, hausner's ratio \& angle of repose and were compressed into tablets using tablet punching machine. Post compression parameters ${ }^{10}$ 
The post compression parameters of the formulated floating tablet weredetermined by carrying out hardness test, friability, weight variation, uniformity of drug content, swelling index \& invitro drug release.

Table 1: Composition of pantoprazole sodium floating tablet

\begin{tabular}{llllllllll}
\hline $\begin{array}{l}\text { Ingredients } \\
(\mathbf{m g})\end{array}$ & FT1 & FT2 & FT3 & FT4 & FT5 & FT6 & FT7 & FT8 & FT9 \\
\hline $\begin{array}{l}\text { Pantoprazole } \\
\text { Sodium }\end{array}$ & 40 & 40 & 40 & 40 & 40 & 40 & 40 & 40 & 40 \\
HPMC K100 & 100 & 75 & 25 & - & - & - & 50 & 75 & 25 \\
HPMC K15 & - & - & - & 100 & 75 & 25 & 50 & 25 & 75 \\
Citric acid & 35 & 35 & 35 & 35 & 35 & 35 & 35 & 35 & 35 \\
NaHCO 3 & 70 & 70 & 70 & 70 & 70 & 70 & 70 & 70 & 70 \\
PVP & 15 & 15 & 15 & 15 & 15 & 15 & 15 & 15 & 15 \\
MCC & 124 & 149 & 174 & 124 & 149 & 174 & 124 & 124 & 124 \\
Talc & 6 & 6 & 6 & 6 & 6 & 6 & 6 & 6 & 6 \\
Mg-stearate & 10 & 10 & 10 & 10 & 10 & 10 & 10 & 10 & 10 \\
Total weight & 400 & 400 & 400 & 400 & 400 & 400 & 400 & 400 & 400 \\
(mg) & & & & & & & & & \\
\hline
\end{tabular}

\section{In vitro drug release studies}

USP dissolution apparatus typeII was employed to study the in vitro drug release from various formulation prepared. The dissolution medium used was $900 \mathrm{ml}$ of acidic buffer of $\mathrm{pH} 1.2$ for $2 \mathrm{~h}$ and phosphate buffer of $\mathrm{pH} 6.8$ for $10 \mathrm{~h}$. the tablet was kept in to the basket. The temperature was maintained at $37^{\circ} \mathrm{C} \pm 0.5^{\circ} \mathrm{C}$ and the stirring rate was $100 \mathrm{rpm}$. Samples were withdrawn at regular time intervals and the same volume was replaced with fresh dissolution medium. The samples were measured by UV-visible spectrophotometer at $283.5 \mathrm{~nm}(\mathrm{pH} 1.2)$ and at $288.7 \mathrm{~nm}(\mathrm{pH} 6.8)$ against a blank. The release studies were conducted in triplicate and the mean values were plotted versus time.

\section{Drug release kinetics of pantoprazole sodium floating tablets}

To analyze the mechanism of drug release and release rate kinetics from the dosage form, the data obtained were fitted into zero order, first order, higuchi release and Korsmeyer and peppas release model.

- $\mathrm{F}=\mathrm{K} . \mathrm{t}$ (Zero-order release kinetics)

- $\mathrm{F}=100\left(1-\mathrm{e}^{-\mathrm{kt}}\right)($ First-order release kinetics)

- $\mathrm{F}=\mathrm{k} \cdot \mathrm{t}^{1 / 2}$ (Higuchi release model)

- $\mathrm{M}_{\mathrm{t}} / \mathrm{M}_{\infty}=\mathrm{K} . \mathrm{t}^{\mathrm{n}}$ (Korsmeyer and peppas release model) 
Where, $F \& M_{t} / M_{\infty}$ arethe fraction of drug release, $K$ is release rate constants, e is exponential coefficient, t release time $\& \mathrm{n}$ is diffusional coefficient.

\section{Accelerated stability study}

Gastro retentive tablets of pantoprazole sodium formulated in the present study were subjected to accelerated stability studies in Aluminum/ Aluminum pouch. The tablets were packed in aluminum pouch and charged for accelerated stability studies at $40^{\circ} \mathrm{C} \pm 2^{\circ} \mathrm{C}$ and $75 \pm 5 \% \mathrm{RH}$ for 3 months in a humidity jar. Floating lag time and drug dissolution profile of exposed sample was carried out.

\section{RESULTS AND DISCUSSION}

\section{Preformulation studies}

Melting point of pantoprazole sodium was found to be $202^{\circ} \mathrm{C}$.

Pantoprazole sodium is soluble in water, phosphate buffer $\mathrm{pH} 7.4$, and ethanol.

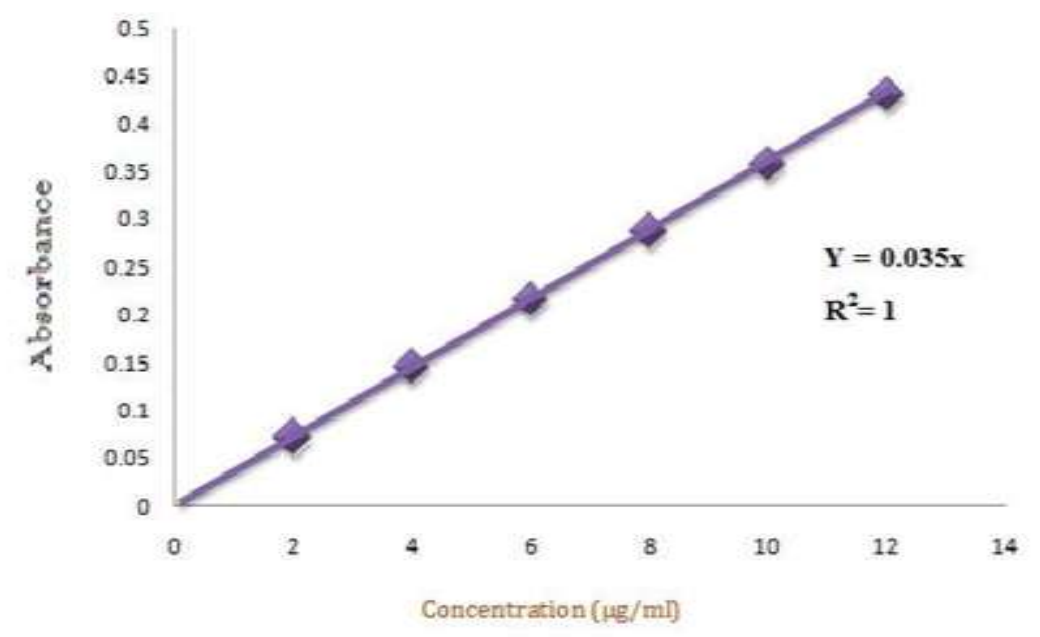

Figure 1: standard calibration curve of pantoprazole sodium in pH6.8 phosphate buffer Compatibility studies

The compatibility studies were determined by the IR spectra of pantoprazole sodium, HPMC K 15, HPMC K100 given in fig 2,3,4, \&5. Hence it indicates that pantoprazole sodium is compatible with the polymers HPMC K15 \& HPMC K100. 


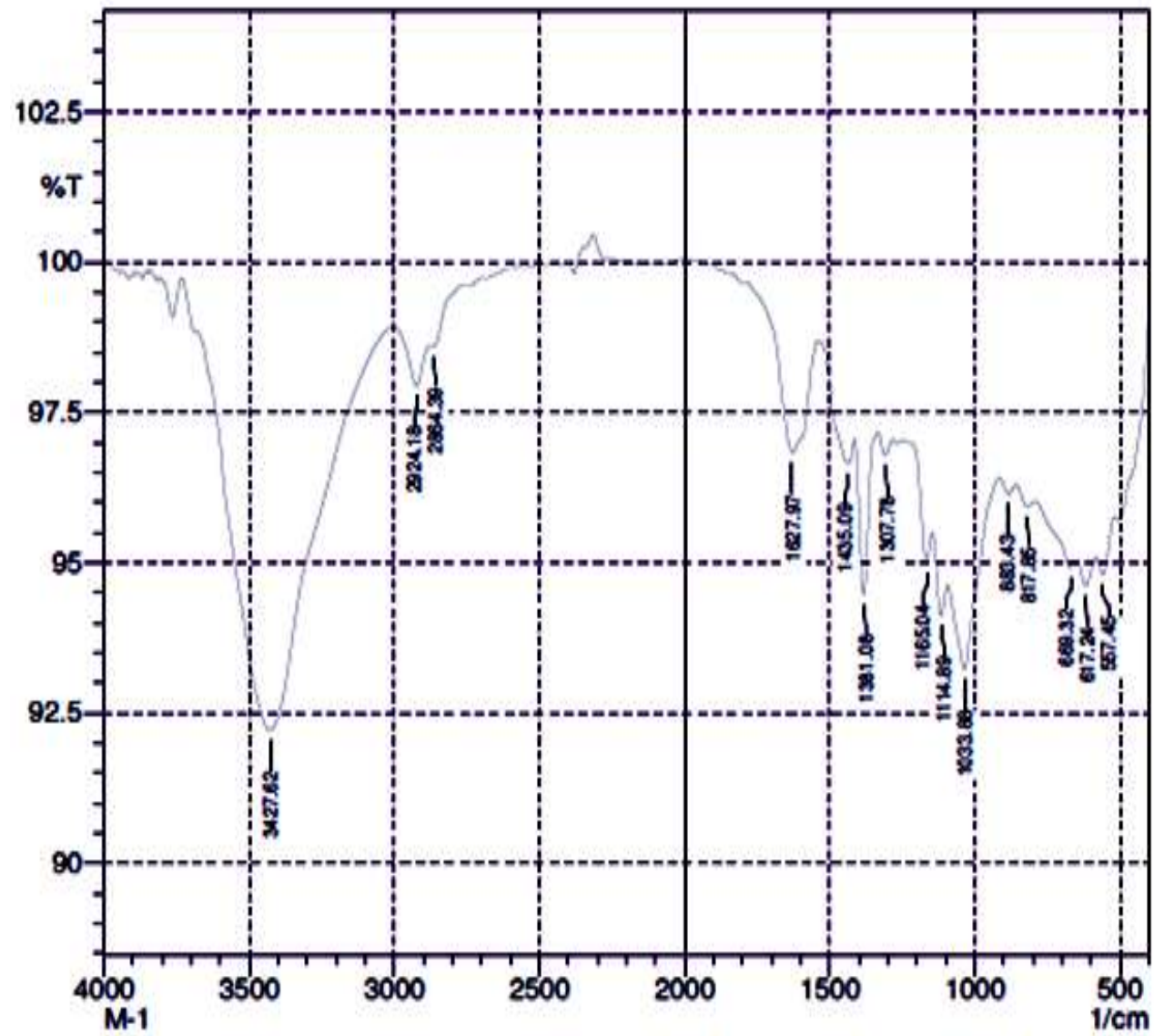

Figure 2: IR spectrum of pantoprazole sodium

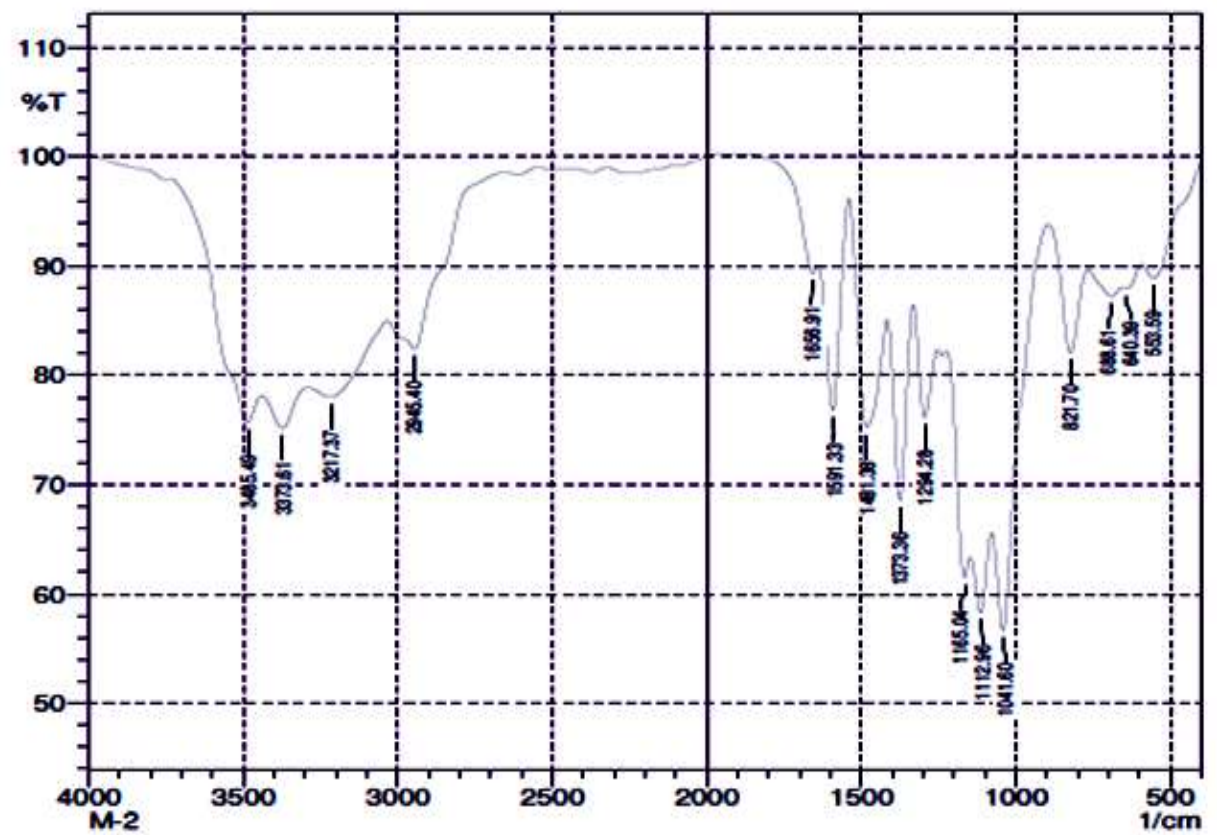

Figure 3: IR spectrum of physical mixture of pantoprazole sodium and HPMC K100 


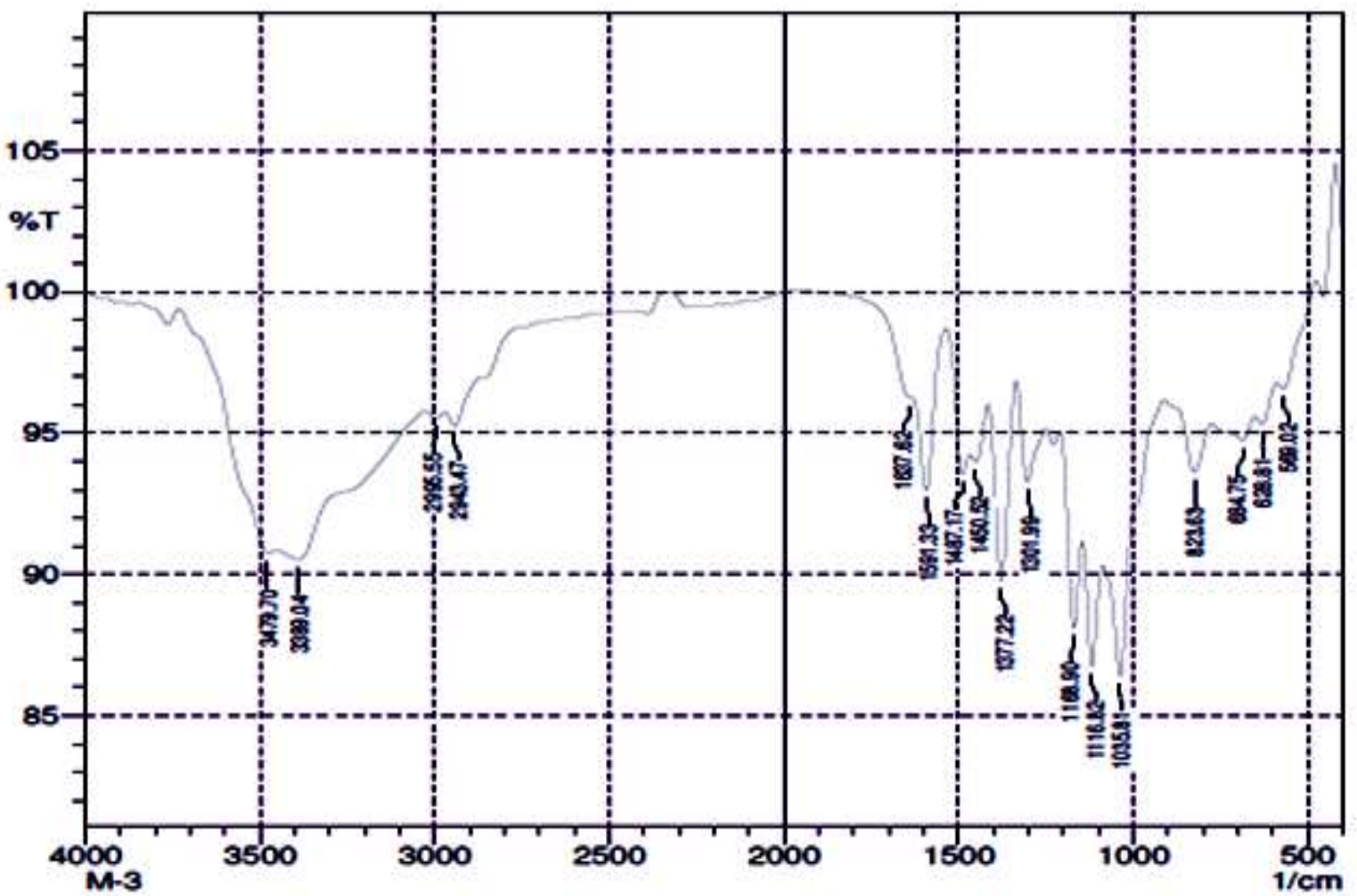

Figure 4: IR spectrum of physical mixture of pantoprazole sodium and HPMC K 15

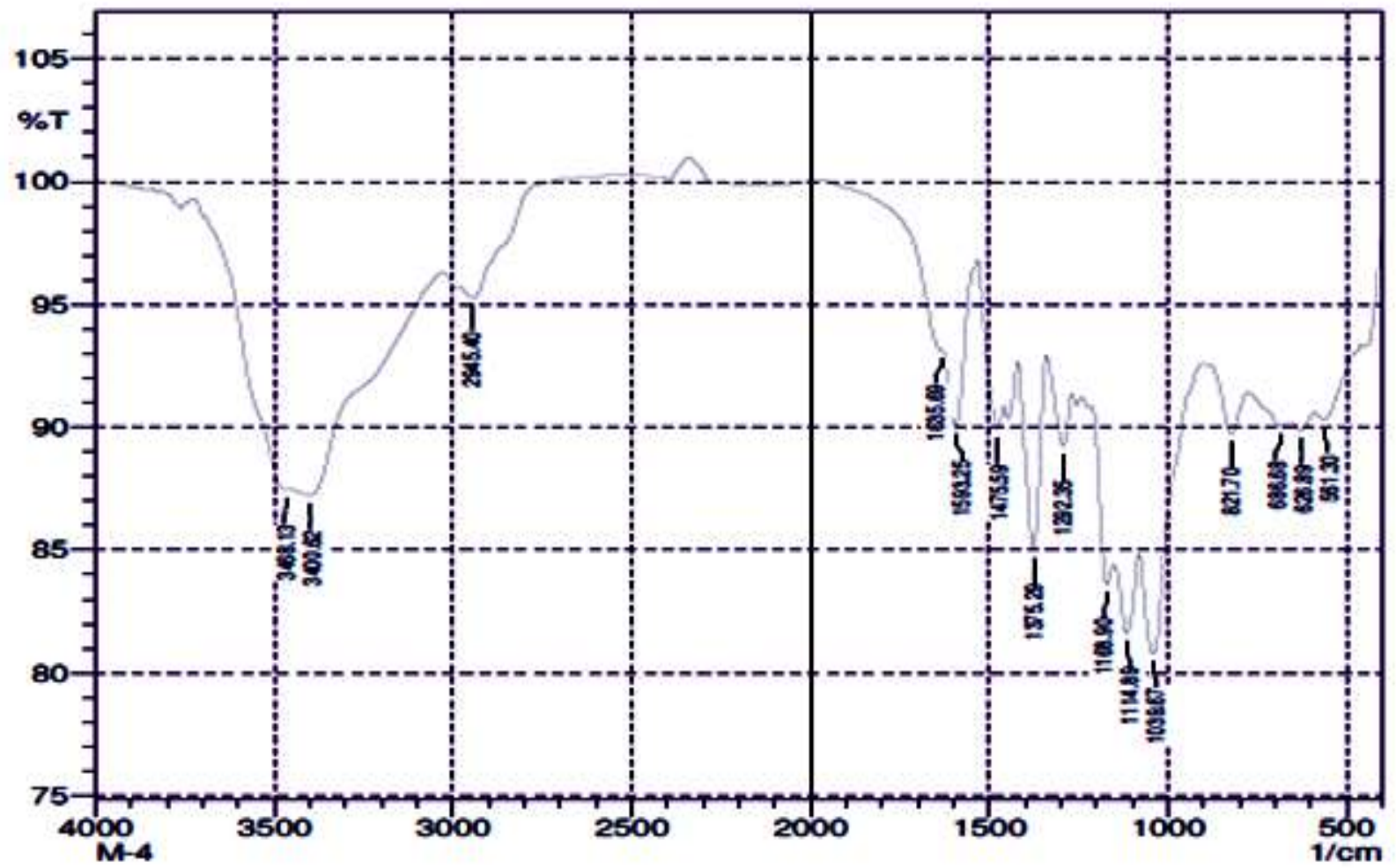

Figure 5: IR spectrum of physical mixture of pantoprazole sodium and HPMC K15 and HPMC K100 


\section{Pre compression parameters}

The various pre compression parameters were performed for 9 different batches of pantoprazole sodium \& HPMC K15 AND HPMC K100. The flow ability was assessed by hausner ratio, carr's index \& angle of repose. Hausner ratio ranged from $1.20 \pm 1.20$ to $1.45 \pm 1.45$ which indicates good flow characteristics. Carr's index was found to be from $16.74 \pm 0.32$ to $27.94 \pm 0.30$ which is an indicative of good flow. Angle of repose ranged from $21.05 \pm 0.02$ to $25.41 \pm 0.37$. Bulk density \& tapped density determined is being listed in table 2 .

\section{Post compression parameters}

The average weight of all formulations ranges from 399 to $404 \mathrm{mg}$ which indicates all the formulations have passed the weight variation test as per the pharmacopeia. The hardness ranged from 4 to $6 \mathrm{~kg} / \mathrm{cm}^{2}$. Formulation FT9 possess good mechanical strength having hardness of $6.0 \pm 0.16$. Friability test was carried out to determine the ability of the tablet to withstand the abrasions during packing \& transportation. The friability of all the formulations ranged from 0.45 to 0.60 which indicates good capability of the tablet since its range should not exceed more than $1 \%$. The other parameters are being listed below in the give table 3 .

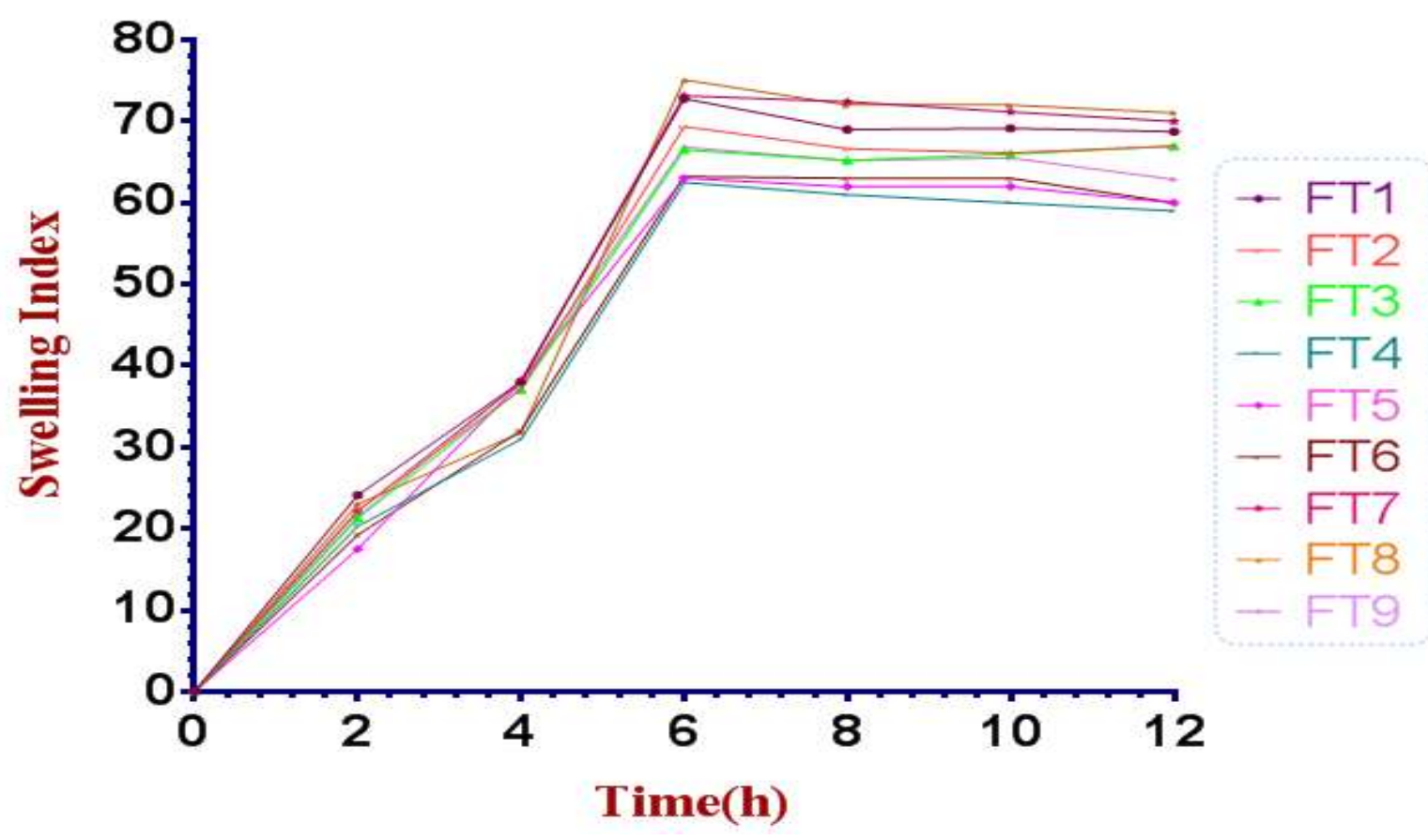

Figure 6: swelling index of the profile of pantoprazole sodium floating tablets 


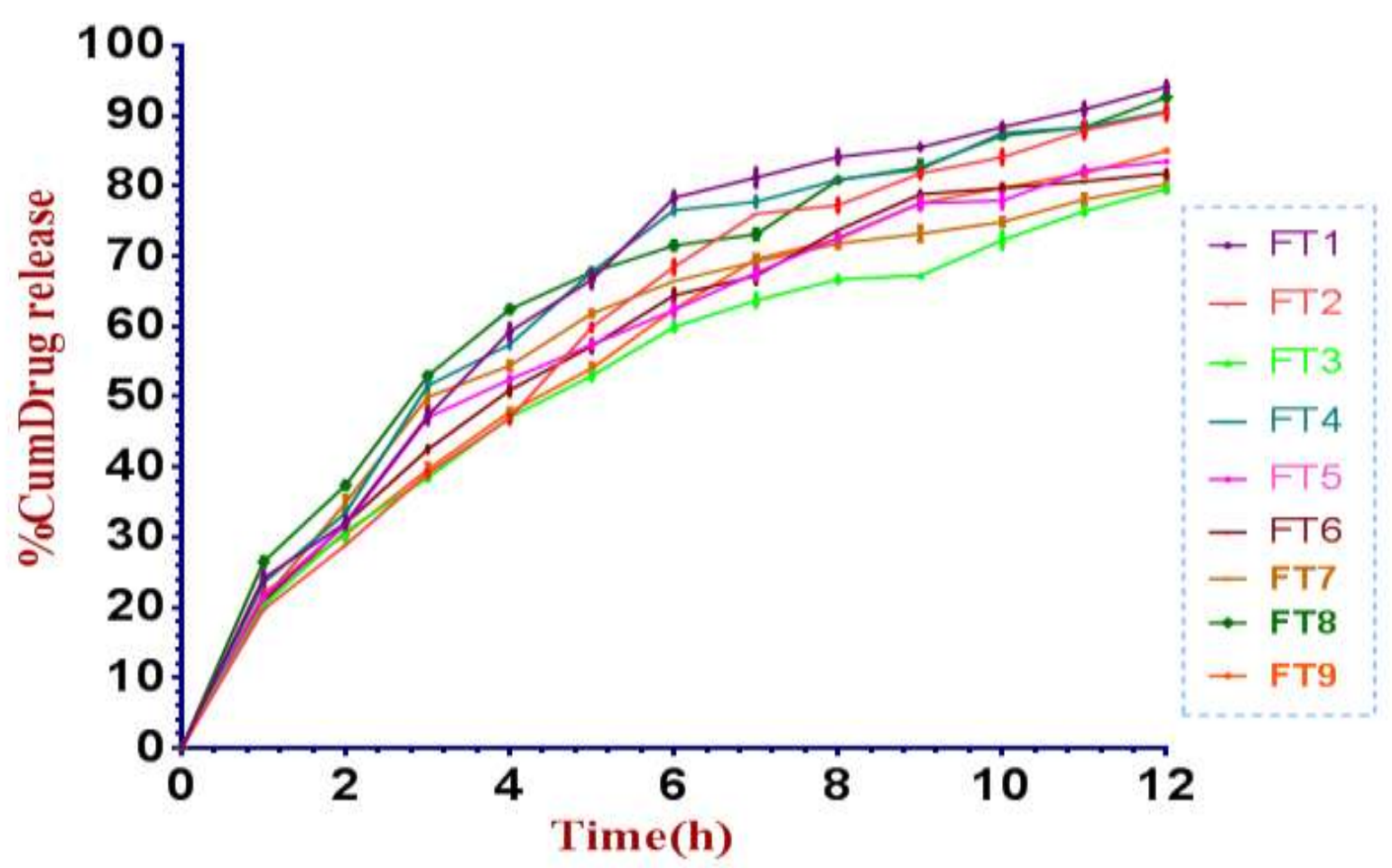

Figure 7: In vitro dissolution profiles for Pantoprazole Sodium Floating tablets

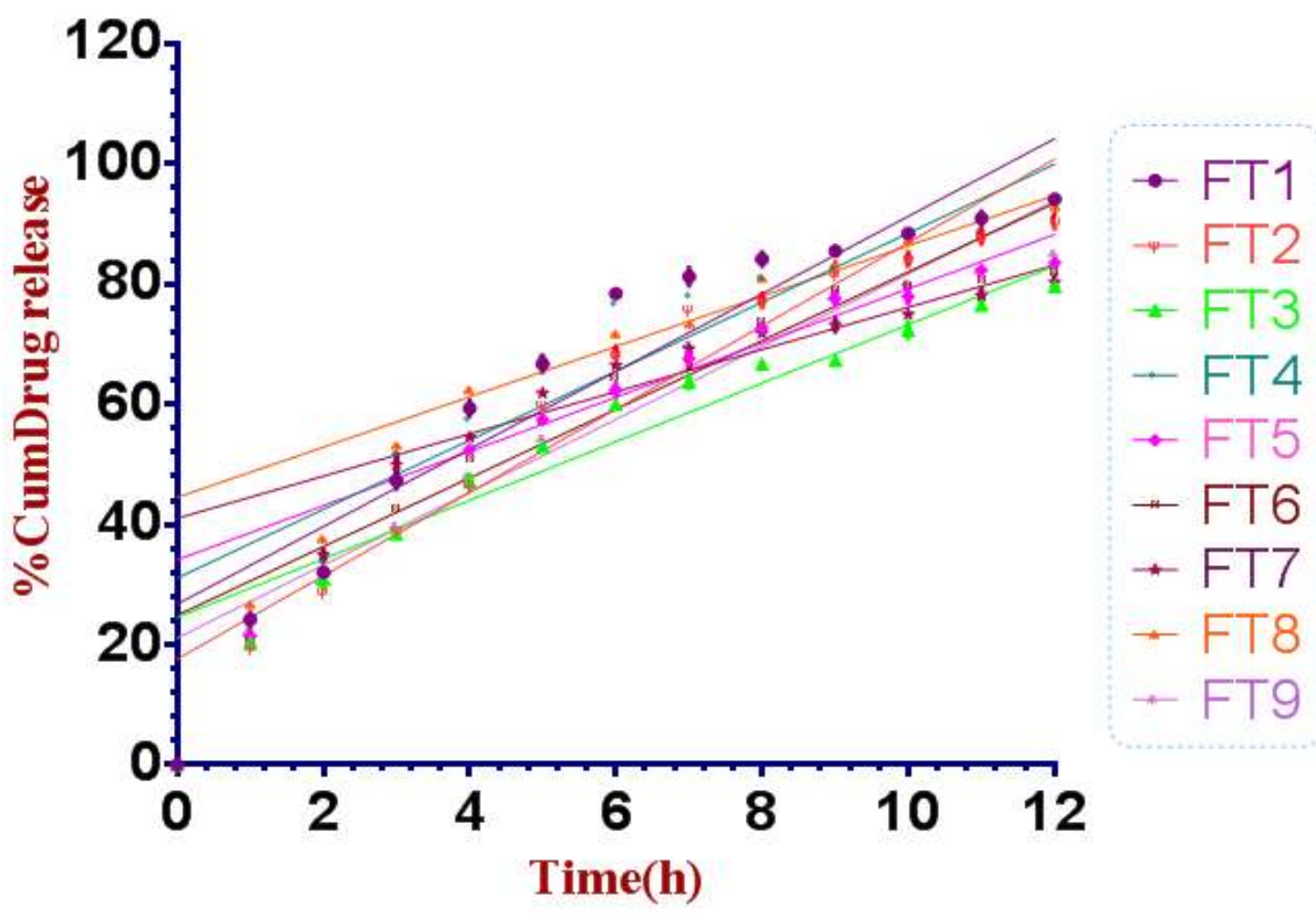

Figure 8: Zero order release kinetic profile of pantoprazole sodium floating tablets 


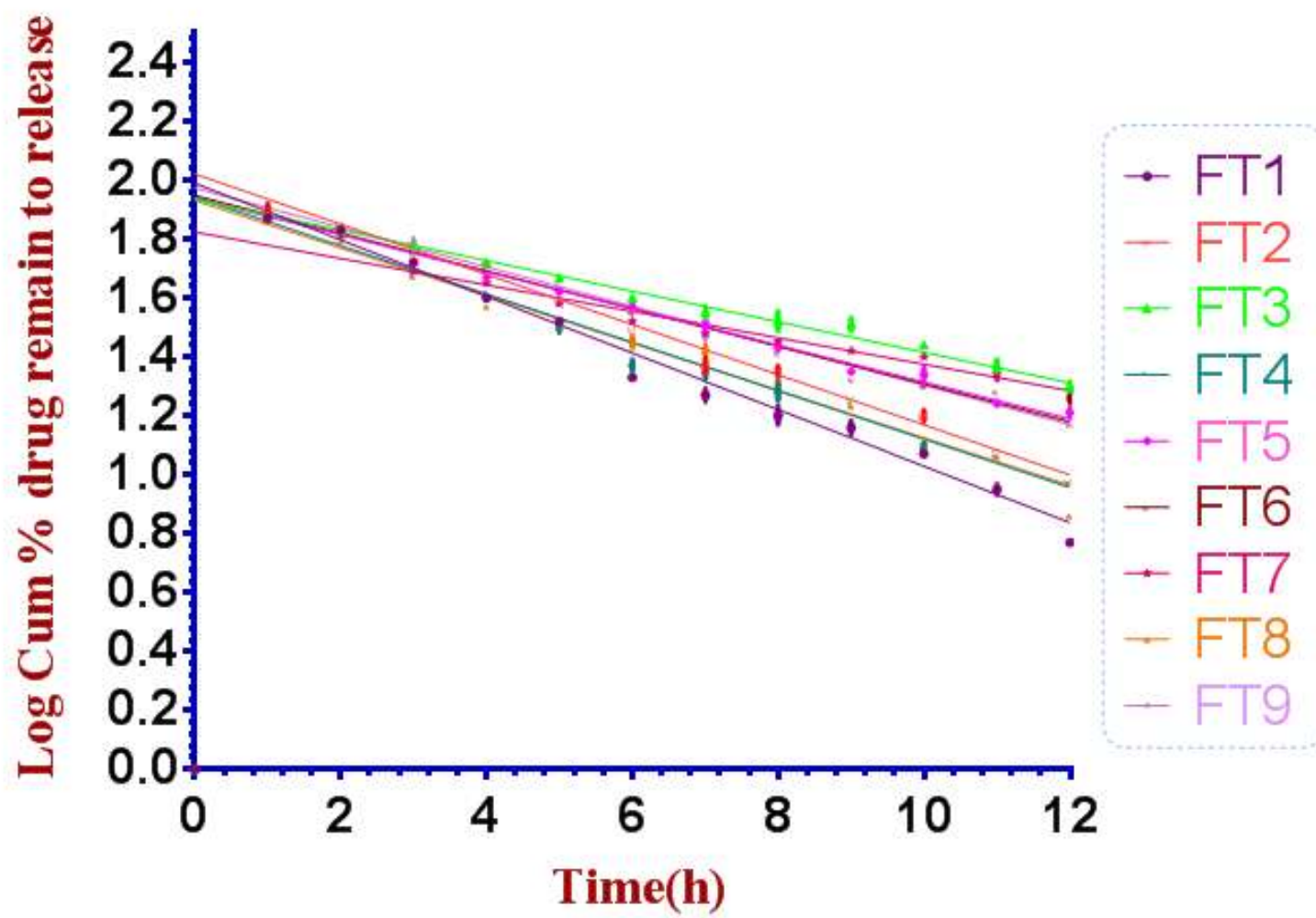

Figure 9: first order release kinetics profile of pantoprazole sodium floating tablets

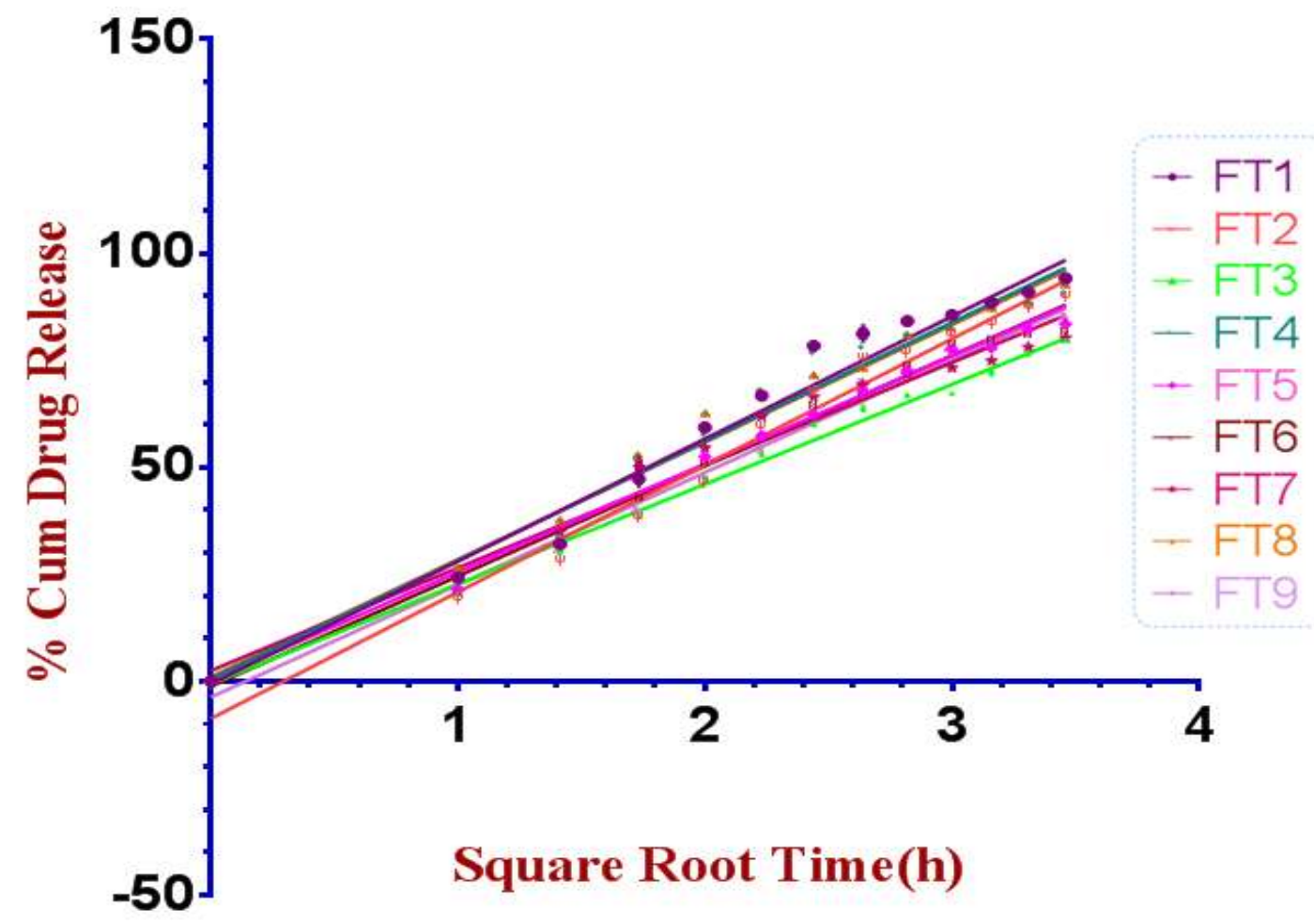

Figure 10: Higuchi's release kinetics profile of pantoprazole sodium floating tablets 


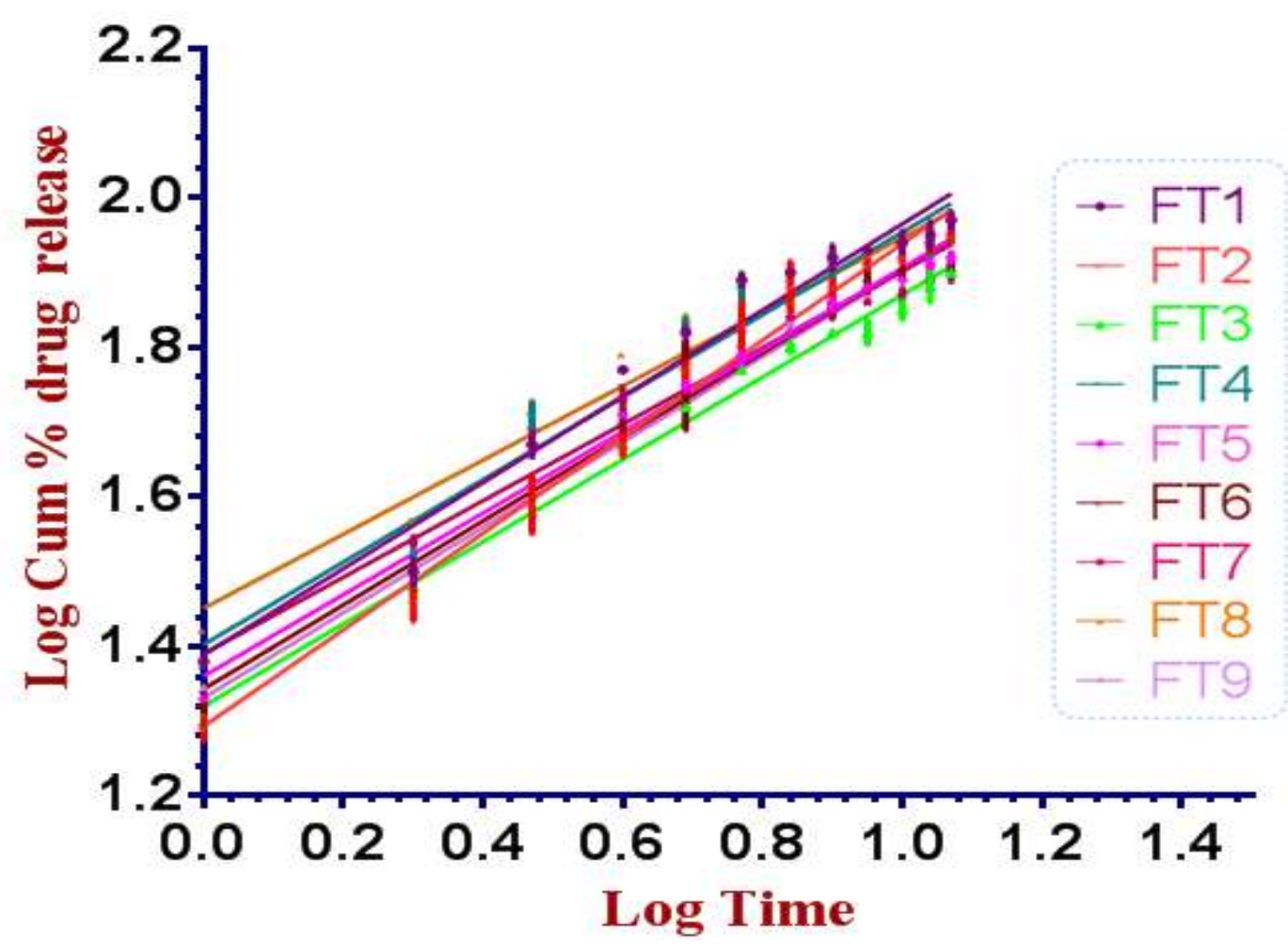

Figure 11: Peppas release kinetics of pantoprazole sodium floating tablets

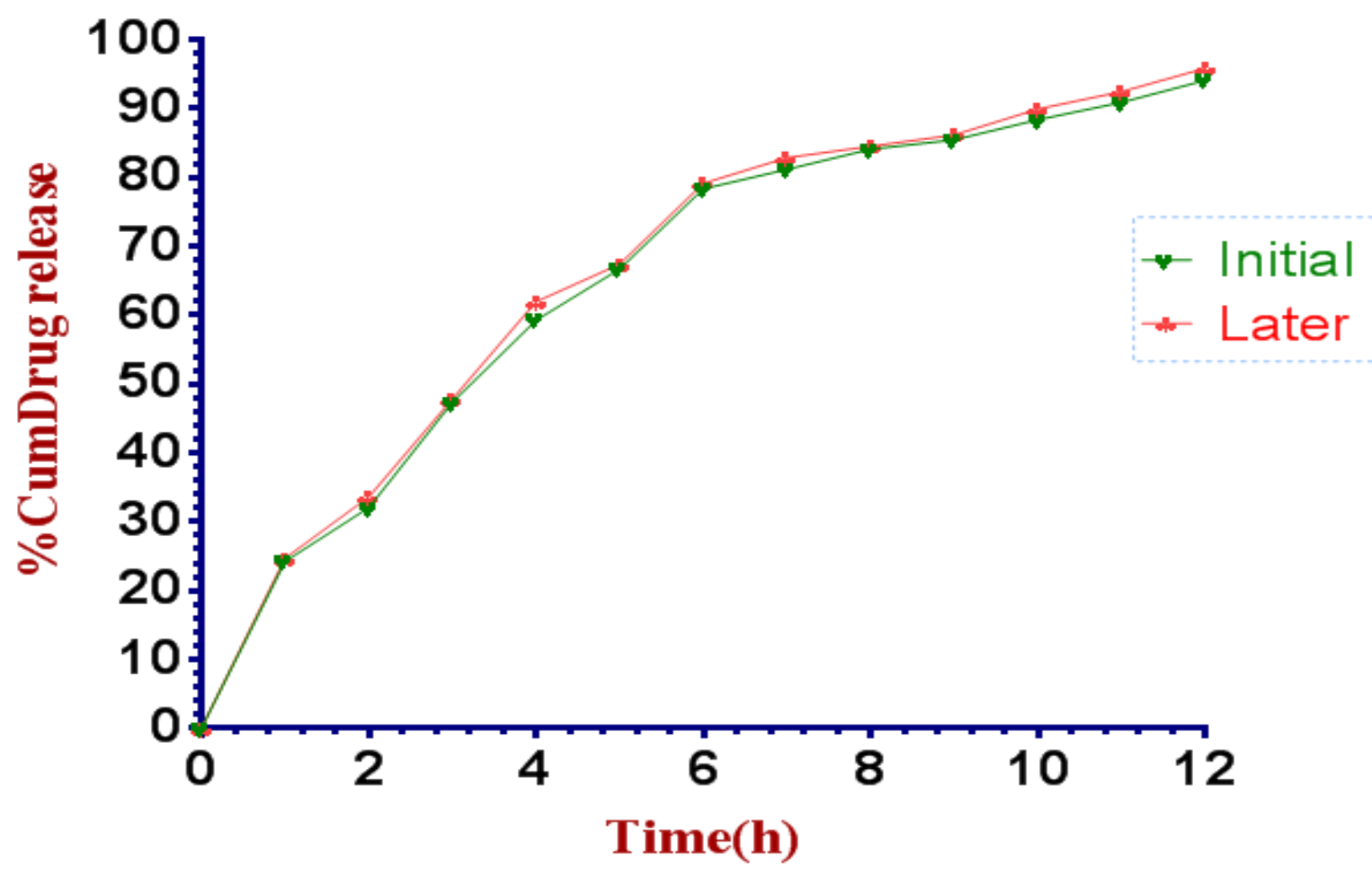

Figure 12: Accelerated stability studies (in vitro release profile of optimized batch (FT1) of pantoprazole sodium floating tablets 
Table 2: Precompression blends of pantoprazole sodium

\begin{tabular}{llllll}
\hline $\begin{array}{l}\text { Batch } \\
\text { code }\end{array}$ & $\begin{array}{l}\text { Angle of } \\
\text { repose } \\
(\boldsymbol{\Theta}) \pm \text { SD }\end{array}$ & $\begin{array}{l}\text { Bulk density } \\
\left(\mathbf{g m} / \mathbf{c m}^{\mathbf{3}}\right) \mathbf{S D}\end{array}$ & $\begin{array}{l}\text { Tapped density } \\
\left(\mathbf{g m} / \mathbf{c m}^{\mathbf{3}}\right) \pm \mathbf{S D}\end{array}$ & $\begin{array}{l}\text { Hausner ratio } \\
(\mathbf{H R}) \pm \text { SD }\end{array}$ & $\begin{array}{l}\text { Carr's } \\
\text { index } \\
(\mathbf{I C}) \pm \text { SD }\end{array}$ \\
\hline FT1 & $22.79 \pm 0.62$ & $0.33 \pm 0.06$ & $0.416 \pm 0.40$ & $1.24 \pm 0.06$ & $19.45 \pm 0.05$ \\
FT2 & $21.05 \pm 0.02$ & $0.33 \pm 0.01$ & $0.414 \pm 0.12$ & $1.20 \pm 1.20$ & $16.74 \pm 0.32$ \\
FT3 & $23.13 \pm 0.26$ & $0.30 \pm 0.02$ & $0.384 \pm 0.26$ & $1.25 \pm 1.26$ & $21.08 \pm 0.21$ \\
FT4 & $24.34 \pm 1.02$ & $0.28 \pm 0.04$ & $0.412 \pm 0.02$ & $1.45 \pm 1.45$ & $31.41 \pm 0.43$ \\
FT5 & $24.58 \pm 0.23$ & $0.34 \pm 0.01$ & $0.510 \pm 0.31$ & $1.45 \pm 1.45$ & $31.20 \pm 0.18$ \\
FT6 & $25.41 \pm 0.37$ & $0.31 \pm 0.03$ & $0.414 \pm 0.29$ & $1.33 \pm 1.45$ & $25.99 \pm 0.12$ \\
FT7 & $22.40 \pm 0.25$ & $0.32 \pm 0.02$ & $0.451 \pm 0.019$ & $1.37 \pm 1.32$ & $27.94 \pm 0.30$ \\
FT8 & $22.17 \pm 0.45$ & $0.34 \pm 0.02$ & $0.421 \pm 0.04$ & $1.23 \pm 1 . .37$ & $19.06 \pm 0.24$ \\
FT9 & $21.29 \pm 0.58$ & $0.4 \pm 0.01$ & $0.504 \pm 0.06$ & $1.26 \pm 1.25$ & $21.03 \pm 0.09$ \\
\hline
\end{tabular}

Table 3: Evaluation of physical parameters of Pantoprazole sodium floating tablets

\begin{tabular}{|c|c|c|c|c|c|c|}
\hline $\begin{array}{l}\text { Batch } \\
\text { code }\end{array}$ & $\begin{array}{l}\text { Weight } \\
\text { variation } \\
\text { Average } \\
\pm \text { SD }\end{array}$ & $\begin{array}{l}\text { Hardness } \\
\left(\mathrm{kg} / \mathrm{cm}^{2}\right) \pm \\
\text { SD }\end{array}$ & $\begin{array}{l}\text { Diameter } \\
(\mathrm{mm}) \pm \text { SD }\end{array}$ & $\begin{array}{l}\text { Thickness } \\
(\mathrm{mm}) \\
\pm \text { SD }\end{array}$ & $\begin{array}{l}\text { Friability } \\
(\%) \\
\pm \text { SD }\end{array}$ & $\begin{array}{l}\text { Drug content } \\
\text { Uniformity } \\
(\%) \pm \text { SD }\end{array}$ \\
\hline FT1 & $404 \pm 1.84$ & $6.0 \pm 0.14$ & $10.70 \pm 0.03$ & $3.0 \pm 0.06$ & $0.50 \pm 0.09$ & $98.44 \pm 0.07$ \\
\hline FT2 & $402 \pm 2.34$ & $5.5 \pm 0.09$ & $11.00 \pm 0.04$ & $2.9 \pm 0.01$ & $0.45 \pm 0.02$ & $98.52 \pm 0.11$ \\
\hline FT3 & $403 \pm 1.68$ & $6.0 \pm 0.12$ & $10.90 \pm 0.12$ & $3.0 \pm 0.04$ & $0.55 \pm 0.05$ & $98.97 \pm 0.04$ \\
\hline FT4 & $399 \pm 1.34$ & $5.0 \pm 0.11$ & $11.02 \pm 0.02$ & $2.9 \pm 0.00$ & $0.50 \pm 0.01$ & $101.01 \pm 0.15$ \\
\hline FT5 & $400 \pm 4.10$ & $4.0 \pm 0.08$ & $11.00 \pm 0.01$ & $3.1 \pm 0.01$ & $0.60 \pm 0.00$ & $98.42 \pm 0.03$ \\
\hline FT6 & $401 \pm 2.10$ & $4.8 \pm 0.08$ & $11.00 \pm 0.01$ & $3.0 \pm 0.01$ & $0.60 \pm 0.00$ & $98.48 \pm 0.21$ \\
\hline FT7 & $402 \pm 1.32$ & $5.5 \pm 0.03$ & $10.90 \pm 0.03$ & $2.9 \pm 0.03$ & $0.50 \pm 0.03$ & $99.12 \pm 0.25$ \\
\hline FT8 & $404 \pm 2.02$ & $5.5 \pm 0.14$ & $11.03 \pm 0.04$ & $3.2 \pm 0.05$ & $0.55 \pm 0.05$ & $98.42 \pm 0.11$ \\
\hline FT9 & $400 \pm 1.23$ & $6.0 \pm 0.16$ & $10.30 \pm 0.01$ & $3.0 \pm 0.02$ & $0.45 \pm 0.02$ & $100.45 \pm 0.26$ \\
\hline
\end{tabular}

Table 4: Swelling index data of floating pantoprazole sodium tablets

\begin{tabular}{llllllllll}
\hline Time (h) & FT1 & FT2 & FT3 & FT4 & FT5 & FT6 & FT7 & FT8 & FT9 \\
\hline 2 & 24.10 & 22.19 & 21.30 & 20.3 & 17.52 & 19.25 & 22.14 & 23.02 & 21.42 \\
4 & 38.05 & 37.16 & 37.10 & 31.0 & 38.0 & 32.03 & 38.10 & 31.80 & 38.03 \\
6 & 72.80 & 69.35 & 66.51 & 62.5 & 63.0 & 63.22 & 73.16 & 75.10 & 66.83 \\
8 & 69.00 & 66.65 & 65.20 & 61.0 & 62.0 & 63.0 & 72.42 & 72.00 & 65.20 \\
10 & 69.15 & 66.14 & 66.00 & 60.0 & 62.0 & 63.0 & 71.16 & 72.00 & 65.52 \\
12 & 68.75 & 66.99 & 66.97 & 59.0 & 60.0 & 60.0 & 70.00 & 71.05 & 62.86 \\
\hline
\end{tabular}

In vitro drug release

Table 5: In vitro drug release data of pantoprazole sodium floating tablets

\begin{tabular}{|c|c|c|c|c|c|}
\hline \multirow[t]{2}{*}{ Time (h) } & \multicolumn{5}{|c|}{ \% Cumulative Release } \\
\hline & FT1 \pm SD & FT2 2 SD & FT3 \pm SD & FT4+SD & FT5 \pm SD \\
\hline 1 & $24.19 \pm 1.05$ & $19.74 \pm 0.15$ & $20.35 \pm 0.15$ & $23.50 \pm 0.60$ & $21.53 \pm 0.50$ \\
\hline 2 & $32.05 \pm 0.95$ & $28.88 \pm 0.30$ & $30.90 \pm 1.55$ & $33.45 \pm 0.55$ & $31.80 \pm 0.53$ \\
\hline 3 & $47.31 \pm 1.25$ & $39.10 \pm 0.49$ & $38.45 \pm 0.47$ & $51.60 \pm .070$ & $47.05 \pm 0.81$ \\
\hline 4 & $59.30 \pm 1.17$ & $46.95 \pm 0.75$ & $47.08 \pm 0.98$ & $57.46 \pm 0.60$ & $52.47 \pm 0.52$ \\
\hline 5 & $66.68 \pm 1.26$ & $59.90 \pm 0.55$ & $52.99 \pm 0.75$ & $67.75 \pm 0.52$ & $57.43 \pm 0.56$ \\
\hline 6 & $78.35 \pm 0.90$ & $68.48 \pm 1.02$ & $59.95 \pm 0.67$ & $76.54 \pm 0.62$ & $62.35 \pm 1.03$ \\
\hline
\end{tabular}




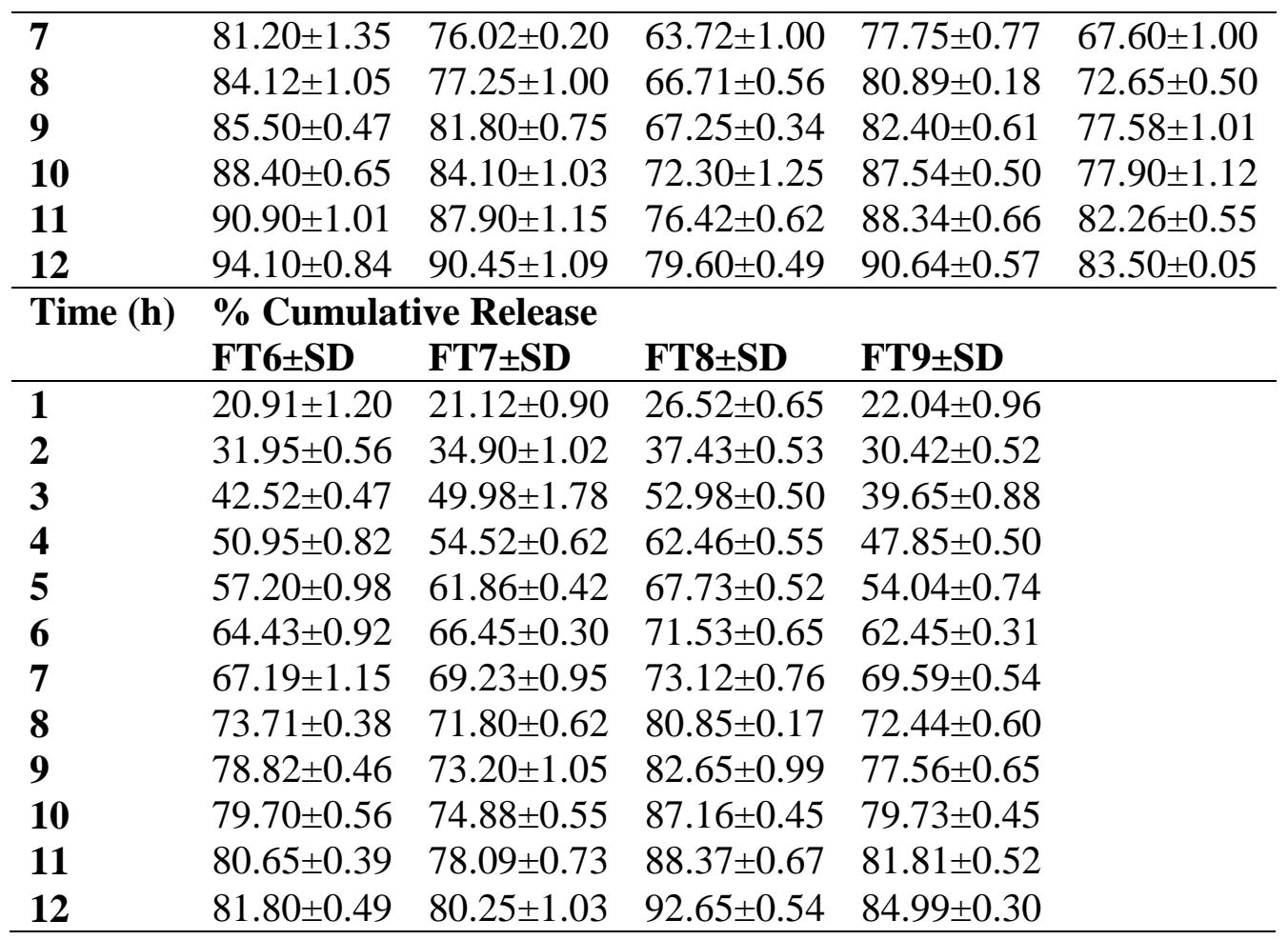

\section{Drug release kinetics}

\section{- Zero order kinetics}

Table 6: Zero order release kinetic data of pantoprazole sodium floating tablets

\begin{tabular}{|c|c|c|c|c|c|}
\hline \multirow[t]{2}{*}{ Time (h) } & \multicolumn{5}{|c|}{ \% Cumulative Release } \\
\hline & FT1 \pm SD & FT2 \pm SD & FT3 \pm SD & FT4+SD & FT5 \pm SD \\
\hline 1 & $24.19 \pm 1.05$ & $19.74 \pm 0.15$ & $20.35 \pm 0.15$ & $23.50 \pm 0.60$ & $21.53 \pm 0.50$ \\
\hline 2 & $32.05 \pm 0.95$ & $28.88 \pm 0.30$ & $30.90 \pm 1.55$ & $33.45 \pm 0.55$ & $31.80 \pm 0.53$ \\
\hline 3 & $47.31 \pm 1.25$ & $39.10 \pm 0.49$ & $38.45 \pm 0.47$ & $51.60 \pm .070$ & $47.05 \pm 0.81$ \\
\hline 4 & $59.30 \pm 1.17$ & $46.95 \pm 0.75$ & $47.08 \pm 0.98$ & $57.46 \pm 0.60$ & $52.47 \pm 0.52$ \\
\hline 5 & $66.68 \pm 1.26$ & $59.90 \pm 0.55$ & $52.99 \pm 0.75$ & $67.75 \pm 0.52$ & $57.43 \pm 0.56$ \\
\hline 6 & $78.35 \pm 0.90$ & $68.48 \pm 1.02$ & $59.95 \pm 0.67$ & $76.54 \pm 0.62$ & $62.35 \pm 1.03$ \\
\hline 7 & $81.20 \pm 1.35$ & $76.02 \pm 0.20$ & $63.72 \pm 1.00$ & $77.75 \pm 0.77$ & $67.60 \pm 1.00$ \\
\hline 8 & $84.12 \pm 1.05$ & $77.25 \pm 1.00$ & $66.71 \pm 0.56$ & $80.89 \pm 0.18$ & $72.65 \pm 0.50$ \\
\hline 9 & $85.50 \pm 0.47$ & $81.80 \pm 0.75$ & $67.25 \pm 0.34$ & $82.40 \pm 0.61$ & $77.58 \pm 1.01$ \\
\hline 10 & $88.40 \pm 0.65$ & $84.10 \pm 1.03$ & $72.30 \pm 1.25$ & $87.54 \pm 0.50$ & $77.90 \pm 1.12$ \\
\hline 11 & $90.90 \pm 1.01$ & $87.90 \pm 1.15$ & $76.42 \pm 0.62$ & $88.34 \pm 0.66$ & $82.26 \pm 0.55$ \\
\hline 12 & $94.10 \pm 0.84$ & $90.45 \pm 1.09$ & $79.60 \pm 0.49$ & $90.64 \pm 0.57$ & $83.50 \pm 0.05$ \\
\hline \multirow[t]{2}{*}{ Time (h) } & \multicolumn{5}{|c|}{ \% Cumulative Release } \\
\hline & FT6 \pm SD & FT7 \pm SD & FT8 \pm SD & FT9 \pm SD & \\
\hline 1 & $20.91 \pm 1.20$ & $21.12 \pm 0.90$ & $26.52 \pm 0.65$ & $22.04 \pm 0.96$ & \\
\hline 2 & $31.95 \pm 0.56$ & $34.90 \pm 1.02$ & $37.43 \pm 0.53$ & $30.42 \pm 0.52$ & \\
\hline 3 & $42.52 \pm 0.47$ & $49.98 \pm 1.78$ & $52.98 \pm 0.50$ & $39.65 \pm 0.88$ & \\
\hline 4 & $50.95 \pm 0.82$ & $54.52 \pm 0.62$ & $62.46 \pm 0.55$ & $47.85 \pm 0.50$ & \\
\hline 5 & $57.20 \pm 0.98$ & $61.86 \pm 0.42$ & $67.73 \pm 0.52$ & $54.04 \pm 0.74$ & \\
\hline 6 & $64.43 \pm 0.92$ & $66.45 \pm 0.30$ & $71.53 \pm 0.65$ & $62.45 \pm 0.31$ & \\
\hline
\end{tabular}




\begin{tabular}{lllll}
\hline $\mathbf{7}$ & $67.19 \pm 1.15$ & $69.23 \pm 0.95$ & $73.12 \pm 0.76$ & $69.59 \pm 0.54$ \\
$\mathbf{8}$ & $73.71 \pm 0.38$ & $71.80 \pm 0.62$ & $80.85 \pm 0.17$ & $72.44 \pm 0.60$ \\
$\mathbf{9}$ & $78.82 \pm 0.46$ & $73.20 \pm 1.05$ & $82.65 \pm 0.99$ & $77.56 \pm 0.65$ \\
$\mathbf{1 0}$ & $79.70 \pm 0.56$ & $74.88 \pm 0.55$ & $87.16 \pm 0.45$ & $79.73 \pm 0.45$ \\
$\mathbf{1 1}$ & $80.65 \pm 0.39$ & $78.09 \pm 0.73$ & $88.37 \pm 0.67$ & $81.81 \pm 0.52$ \\
$\mathbf{1 2}$ & $81.80 \pm 0.49$ & $80.25 \pm 1.03$ & $92.65 \pm 0.54$ & $84.99 \pm 0.30$
\end{tabular}

\section{- First order release kinetics}

Table 7: First order release kinetic data of pantoprazole sodium floating tablets

\begin{tabular}{|c|c|c|c|c|c|}
\hline \multirow[t]{2}{*}{ Time (h) } & \multicolumn{5}{|c|}{ log Cumulative \% drug remain to release } \\
\hline & FT1 1 SD & FT2 \pm SD & FT3 \pm SD & FT4 4 SD & FT5 \pm SD \\
\hline 1 & $1.87 \pm 0.005$ & $1.90 \pm 0.017$ & $1.90 \pm 0.007$ & $1.88 \pm 0.007$ & $1.89 \pm 0.012$ \\
\hline 2 & $1.83 \pm 0.008$ & $1.85 \pm 0.012$ & $1.83 \pm 0.008$ & $1.82 \pm 0.010$ & $1.83 \pm 0.011$ \\
\hline 3 & $1.72 \pm 0.007$ & $1.78 \pm 0.005$ & $1.78 \pm 0.008$ & $1.68 \pm 0.009$ & $1.72 \pm 0.005$ \\
\hline 4 & $1.60 \pm 0.010$ & $1.72 \pm 0.011$ & $1.72 \pm 0.0 \backslash \backslash 08$ & $1.62 \pm 0.012$ & $1.67 \pm 0.008$ \\
\hline 5 & $1.52 \pm 0.009$ & $1.60 \pm 0.009$ & $1.67 \pm 0.004$ & $1.50 \pm 0.014$ & $1.62 \pm 0.009$ \\
\hline 6 & $1.33 \pm 0.012$ & $1.49 \pm 0.005$ & $1.60 \pm 0.003$ & $1.37 \pm 0.020$ & $1.57 \pm 0.007$ \\
\hline 7 & $1.27 \pm 0.018$ & $1.37 \pm 0.025$ & $1.55 \pm 0.017$ & $1.34 \pm 0.016$ & $1.51 \pm 0.017$ \\
\hline 8 & $1.20 \pm 0.029$ & $1.35 \pm 0.016$ & $1.52 \pm 0.032$ & $1.28 \pm 0.029$ & $1.43 \pm 0.008$ \\
\hline 9 & $1.16 \pm 0.021$ & $1.26 \pm 0.009$ & $1.51 \pm 0.019$ & $1.24 \pm 0.008$ & $1.35 \pm 0.009$ \\
\hline 10 & $1.07 \pm 0.008$ & $1.20 \pm 0.017$ & $1.44 \pm 0.002$ & $1.09 \pm 0.015$ & $1.34 \pm 0.021$ \\
\hline 11 & $0.95 \pm 0.015$ & $1.08 \pm 0.009$ & $1.37 \pm 0.020$ & $1.06 \pm 0.007$ & $1.24 \pm 0.007$ \\
\hline & $0 . .77 \pm 0.006$ & $1.98 \pm 0.007$ & $1.30 \pm 0.014$ & $0.97 \pm 0.008$ & $1.21 \pm 0.015$ \\
\hline \multirow[t]{2}{*}{ Time (h) } & \multicolumn{5}{|c|}{ log Cumulative \% drug remain to release } \\
\hline & FT6 \pm SD & FT7 \pm SD & FT8 \pm SD & FT9 \pm SD & \\
\hline 1 & $1.89 \pm 0.002$ & $1.89 \pm 0.004$ & $1.86 \pm 0.007$ & $1.89 \pm 0.004$ & \\
\hline 2 & $1.83 \pm 0.006$ & $1.81 \pm 0.002$ & $1.79 \pm 0.010$ & $1.84 \pm 0.012$ & \\
\hline 3 & $1.75 \pm 0.007$ & $1.69 \pm 0.004$ & $1.67 \pm 0.009$ & $1.78 \pm 0.018$ & \\
\hline 4 & $1.69 \pm 0.011$ & $1.65 \pm 0.002$ & $1.57 \pm 0.012$ & $1.71 \pm 0.003$ & \\
\hline 5 & $1.63 \pm 0.002$ & $1.58 \pm 0.006$ & $1.50 \pm 0.014$ & $1.66 \pm 0.005$ & \\
\hline 6 & $1.55 \pm 0.007$ & $1.52 \pm 0.007$ & $1.45 \pm 0.020$ & $1.57 \pm 0.017$ & \\
\hline 7 & $1.51 \pm 0.009$ & $1.48 \pm 0.002$ & $1.42 \pm 0.016$ & $1.48 \pm 0.020$ & \\
\hline 8 & $1.41 \pm 0.010$ & $1.45 \pm 0.003$ & $1.29 \pm 0.029$ & $1.44 \pm 0.015$ & \\
\hline 9 & $1.32 \pm 0.013$ & $1.42 \pm 0.010$ & $1.23 \pm 0.008$ & $1.35 \pm 0.005$ & \\
\hline 10 & $1.30 \pm 0.009$ & $1.40 \pm 0.004$ & $1.10 \pm 0.015$ & $1.30 \pm 0.007$ & \\
\hline 11 & $1.28 \pm 0.002$ & $1.34 \pm 0.017$ & $1.06 \pm 0.006$ & $1.25 \pm 0.006$ & \\
\hline 12 & $1.26 \pm 0.014$ & $1.29 \pm 0.009$ & $0.86 \pm 0.008$ & $1.17 \pm 0.012$ & \\
\hline
\end{tabular}

\section{Higuchi Model}

Table 8: Higuchi's release kinetic data of pantoprazole sodium floating tablets

\begin{tabular}{llllll}
\hline \multirow{2}{*}{ Square root time (h) } & \multicolumn{2}{c}{ Cumulative \% Drug Release } & & \\
& FT1 \pm SD & FT2 \pm SD & FT3 \pm SD & FT4 \pm SD & FT5 \pm SD \\
\hline $\mathbf{1}$ & $24.19 \pm 1.05$ & $19.74 \pm 0.15$ & $20.35 \pm 0.15$ & $23.50 \pm 0.60$ & $21.53 \pm 0.50$ \\
$\mathbf{1 . 4 1 4}$ & $32.05 \pm 0.95$ & $28.88 \pm 0.30$ & $30.90 \pm 1.55$ & $33.45 \pm 0.55$ & $31.80 \pm 0.53$ \\
$\mathbf{1 . 7 3 2}$ & $47.31 \pm 1.25$ & $39.10 \pm 0.49$ & $38.45 \pm 0.47$ & $51.60 \pm .070$ & $47.05 \pm 0.81$ \\
$\mathbf{2}$ & $59.30 \pm 1.17$ & $46.95 \pm 0.75$ & $47.08 \pm 0.98$ & $57.46 \pm 0.60$ & $52.47 \pm 0.52$ \\
$\mathbf{2 . 2 3}$ & $66.68 \pm 1.26$ & $59.90 \pm 0.55$ & $52.99 \pm 0.75$ & $67.75 \pm 0.52$ & $57.43 \pm 0.56$ \\
$\mathbf{2 . 4 4}$ & $78.35 \pm 0.90$ & $68.48 \pm 1.02$ & $59.95 \pm 0.67$ & $76.54 \pm 0.62$ & $62.35 \pm 1.03$ \\
\hline
\end{tabular}




\begin{tabular}{llllll}
\hline $\mathbf{2 . 6 4}$ & $81.20 \pm 1.35$ & $76.02 \pm 0.20$ & $63.72 \pm 1.00$ & $77.75 \pm 0.77$ & $67.60 \pm 1.00$ \\
$\mathbf{2 . 8 2}$ & $84.12 \pm 1.05$ & $77.25 \pm 1.00$ & $66.71 \pm 0.56$ & $80.89 \pm 0.18$ & $72.65 \pm 0.50$ \\
$\mathbf{3}$ & $85.50 \pm 0.47$ & $81.80 \pm 0.75$ & $67.25 \pm 0.34$ & $82.40 \pm 0.61$ & $77.58 \pm 1.01$ \\
$\mathbf{3 . 1 6}$ & $88.40 \pm 0.65$ & $84.10 \pm 1.03$ & $72.30 \pm 1.25$ & $87.54 \pm 0.50$ & $77.90 \pm 1.12$ \\
$\mathbf{3 . 3 1}$ & $90.90 \pm 1.01$ & $87.90 \pm 1.15$ & $76.42 \pm 0.62$ & $88.34 \pm 0.66$ & $82.26 \pm 0.55$ \\
$\mathbf{3 . 4 6}$ & $94.10 \pm 0.84$ & $90.45 \pm 1.09$ & $79.60 \pm 0.49$ & $90.64 \pm 0.57$ & $83.50 \pm 0.05$ \\
\hline
\end{tabular}

Square root time (h) Cumulative \% Drug Release

FT6 \pm SD $\quad$ FT7 \pm SD $\quad$ FT8 \pm SD $\quad$ FT9 \pm SD

\begin{tabular}{lllll}
\hline $\mathbf{1}$ & $20.91 \pm 1.20$ & $21.12 \pm 0.90$ & $26.52 \pm 0.65$ & $22.04 \pm 0.96$ \\
$\mathbf{1 . 4 1 4}$ & $31.95 \pm 0.56$ & $21.90 \pm 1.02$ & $37.43 \pm 0.53$ & $30.42 \pm 0.52$ \\
$\mathbf{1 . 7 3 2}$ & $42.52 \pm 0.47$ & $23.65 \pm 1.78$ & $52.98 \pm 0.50$ & $39.65 \pm 0.88$ \\
$\mathbf{2}$ & $50.95 \pm 0.82$ & $30.52 \pm 0.62$ & $62.46 \pm 0.55$ & $47.85 \pm 0.50$ \\
$\mathbf{2 . 2 3}$ & $57.20 \pm 0.98$ & $37.68 \pm 0.42$ & $67.73 \pm 0.52$ & $54.04 \pm 0.74$ \\
$\mathbf{2 . 4 4}$ & $64.43 \pm 0.92$ & $42.45 \pm 0.30$ & $71.53 \pm 0.65$ & $62.45 \pm 0.31$ \\
$\mathbf{2 . 6 4}$ & $67.19 \pm 1.15$ & $52.23 \pm 0.95$ & $73.12 \pm 0.76$ & $69.59 \pm 0.54$ \\
$\mathbf{2 . 8 2}$ & $73.71 \pm 0.38$ & $61.80 \pm 0.62$ & $80.85 \pm 0.17$ & $72.44 \pm 0.60$ \\
$\mathbf{3}$ & $78.82 \pm 0.46$ & $71.20 \pm 1.05$ & $82.65 \pm 0.99$ & $77.56 \pm 0.65$ \\
$\mathbf{3 . 1 6}$ & $79.70 \pm 0.56$ & $72.88 \pm 0.55$ & $87.16 \pm 0.45$ & $79.73 \pm 0.45$ \\
$\mathbf{3 . 3 1}$ & $80.65 \pm 0.39$ & $74.09 \pm 0.73$ & $88.37 \pm 0.67$ & $81.81 \pm 0.52$ \\
$\mathbf{3 . 4 6}$ & $81.80 \pm 0.49$ & $82.25 \pm 1.03$ & $92.65 \pm 0.54$ & $84.99 \pm 0.30$
\end{tabular}

Korsmeyer-Peppas Model

Table 9: Peppas release kinetics data of pantoprazole sodium floating tablets

\begin{tabular}{llllll}
\hline Log & \multicolumn{2}{l}{ \% log Cumulative Release } & & \\
Time (h) & FT1 \pm SD & FT2 \pm SD & FT3 \pm SD & FT4 \pm SD & FT5 \pm SD \\
\hline $\mathbf{0}$ & $1.38 \pm 0.006$ & $1.29 \pm 0.015$ & $1.30 \pm 0.006$ & $1.37 \pm 0.003$ & $1.33 \pm 0.005$ \\
$\mathbf{0 . 3 0}$ & $1.50 \pm 0.017$ & $1.46 \pm 0.025$ & $1.48 \pm 0.017$ & $1.52 \pm 0.008$ & $1.50 \pm 0.008$ \\
$\mathbf{0 . 4 7}$ & $1.67 \pm 0.015$ & $1.59 \pm 0.038$ & $1.58 \pm 0.004$ & $1.71 \pm 0.017$ & $1.67 \pm 0.015$ \\
$\mathbf{0 . 6 0}$ & $1.77 \pm 0.005$ & $1.67 \pm 0.015$ & $1.67 \pm 0.008$ & $1.75 \pm 0.002$ & $1.71 \pm 0.002$ \\
$\mathbf{0 . 6 9}$ & $1.82 \pm 0.006$ & $1.77 \pm 0.009$ & $1.72 \pm 0.001$ & $1.83 \pm 0.007$ & $1.75 \pm 0.007$ \\
$\mathbf{0 . 7 7}$ & $1.89 \pm 0.008$ & $1.83 \pm 0.031$ & $1.77 \pm 0.004$ & $1.88 \pm 0.017$ & $1.79 \pm 0.017$ \\
$\mathbf{0 . 8 4}$ & $1.90 \pm 0.005$ & $1.88 \pm 0.034$ & $1.80 \pm 0.007$ & $1.89 \pm 0.005$ & $1.82 \pm 0.005$ \\
$\mathbf{0 . 9 0}$ & $1.92 \pm 0.016$ & $1.88 \pm 0.009$ & $1.82 \pm 0.001$ & $1.90 \pm 0.012$ & $1.86 \pm 0.012$ \\
$\mathbf{0 . 9 5}$ & $1.93 \pm 0.005$ & $1.91 \pm 0.007$ & $1.82 \pm 0.016$ & $1.91 \pm 0.004$ & $1.88 \pm 0.004$ \\
$\mathbf{1}$ & $1.94 \pm 0.015$ & $1.92 \pm 0.007$ & $1.85 \pm 0.012$ & $1.94 \pm 0.004$ & $1.89 \pm 0.004$ \\
$\mathbf{1 . 0 4}$ & $1.95 \pm 0.017$ & $1.94 \pm 0.013$ & $1.88 \pm 0.019$ & $1.94 \pm 0.016$ & $1.91 \pm 0.016$ \\
$\mathbf{1 . 0 7}$ & $1.97 \pm 0.011$ & $1.95 \pm 0.012$ & $1.90 \pm 0.005$ & $1.95 \pm 0.006$ & $1.92 \pm 0.006$ \\
\hline Log & \% log Cumulative Release & & & \\
Time (h) & FT6 \pm SD & FT7 \pm SD & FT8 \pm SD & FT9 \pm SD & \\
\cline { 1 - 1 } & $1.32 \pm 0.017$ & $1.32 \pm 0.005$ & $1.42 \pm 0.004$ & $1.34 \pm 0.006$ & \\
$\mathbf{0 . 3 0}$ & $1.50 \pm 0.002$ & $1.54 \pm 0.007$ & $1.57 \pm 0.003$ & $1.48 \pm 0.017$ & \\
$\mathbf{0 . 4 7}$ & $1.62 \pm 0.004$ & $1.69 \pm 0.012$ & $1.72 \pm 0.006$ & $1.59 \pm 0.004$ & \\
$\mathbf{0 . 6 0}$ & $1.70 \pm 0.015$ & $1.73 \pm 0.016$ & $1.79 \pm 0.005$ & $1.67 \pm 0.008$ & \\
$\mathbf{0 . 6 9}$ & $1.75 \pm 0.060$ & $1.79 \pm 0.013$ & $1.83 \pm 0.012$ & $1.73 \pm 0.001$ & \\
$\mathbf{0 . 7 7}$ & $1.80 \pm 0.006$ & $1.82 \pm 0.007$ & $1.85 \pm 0.003$ & $1.79 \pm 0.007$ & \\
$\mathbf{0 . 8 4}$ & $1.82 \pm 0.004$ & $1.84 \pm 0.003$ & $1.86 \pm 0.005$ & $1.84 \pm 0.016$ & \\
$\mathbf{0 . 9 0}$ & $1.86 \pm 0.016$ & $1.85 \pm 0.011$ & $1.90 \pm 0.014$ & $1.85 \pm 0.012$ & \\
\hline & & \multicolumn{3}{c}{} & \\
& & & &
\end{tabular}




\begin{tabular}{lllll}
\hline $\mathbf{0 . 9 5}$ & $1.89 \pm 0.015$ & $1.86 \pm 0.002$ & $1.91 \pm 0.014$ & $1.88 \pm 0.019$ \\
$\mathbf{1}$ & $1.90 \pm 0.007$ & $1.87 \pm 0.009$ & $1.94 \pm 0.007$ & $1.90 \pm 0.005$ \\
$\mathbf{1 . 0 4}$ & $1.90 \pm 0.002$ & $1.89 \pm 0.007$ & $1.94 \pm 0.016$ & $1.91 \pm 0.008$ \\
$\mathbf{1 . 0 7}$ & $1.91 \pm 0.013$ & $1.90 \pm 0.012$ & $1.96 \pm 0.003$ & $1.92 \pm 0.006$ \\
\hline
\end{tabular}

Table 10: Regression coefficients fit to different drug release kinetic models for pantoprazole sodium floating tablet

\begin{tabular}{llllll}
\hline Formulation code & $\begin{array}{l}\text { Zero order } \\
\mathbf{r}^{\mathbf{2}}\end{array}$ & $\begin{array}{l}\mathbf{1}^{\text {st }} \text { order } \\
\mathbf{r}^{\mathbf{2}}\end{array}$ & $\begin{array}{l}\text { Higuchi } \\
\mathbf{r}^{\mathbf{2}}\end{array}$ & $\begin{array}{l}\text { Peppas } \\
\mathbf{r}^{\mathbf{2}}\end{array}$ & $\mathbf{n}$ \\
\hline FT1 & 0.9045 & 0.9865 & 0.9582 & 0.9656 & 0.5760 \\
FT2 & 0.9360 & 0.9924 & 0.9795 & 0.6453 & 0.9848 \\
FT3 & 0.9464 & 0.9899 & 0.9889 & 0.9905 & 0.5499 \\
FT4 & 0.8803 & 0.9865 & 0.9550 & 0.9593 & 0.5512 \\
FT5 & 0.9300 & 0.9929 & 0.9838 & 0.9823 & 0.5481 \\
FT6 & 0.9238 & 0.9808 & 0.9800 & 0.9882 & 0.5619 \\
FT7 & 0.8587 & 0.9591 & 0.9318 & 0.9473 & 0.5115 \\
FT8 & 0.9105 & 0.9840 & 0.9730 & 0.9732 & 0.5071 \\
FT9 & 0.9548 & 0.9966 & 0.9904 & 0.9930 & 0.5705 \\
\hline Accel
\end{tabular}

Table 11: Accelerated stability studies (in vitro release) data of optimized batch (FT1) of pantoprazole floating tablet

\begin{tabular}{lll}
\hline Time ( h ) & Initial & After 3 months \\
\hline 1 & $24.19 \pm 1.05$ & $24.65 \pm 0.53$ \\
2 & $32.05 \pm 0.95$ & $33.60 \pm 0.30$ \\
3 & $47.31 \pm 1.25$ & $47.82 \pm 0.12$ \\
4 & $59.30 \pm 1.17$ & $61.98 \pm 0.51$ \\
5 & $66.68 \pm 1.26$ & $67.38 \pm 0.77$ \\
6 & $78.35 \pm 0.90$ & $79.15 \pm 0.30$ \\
7 & $81.20 \pm 1.35$ & $82.89 \pm 0.54$ \\
8 & $84.12 \pm 1.05$ & $84.52 \pm 1.21$ \\
9 & $85.50 \pm 0.47$ & $86.19 \pm 0.78$ \\
10 & $88.40 \pm 0.65$ & $89.99 \pm 1.02$ \\
11 & $90.90 \pm 1.01$ & $92.45 \pm 0.32$ \\
12 & $94.10 \pm 0.84$ & $95.89 \pm 0.57$ \\
\hline
\end{tabular}

\section{CONCLUSION}

Formulation and evaluation of floating tablets of pantoprazole sodium was carried out successfully. HPMC K 15, HPMC K100 were compatible with pantoprazole sodium and thus suitable for the formulation of pantoprazole sodium floating tablets. With the present study it was concluded that, floating tablet of pantoprazole sodium can increase the gastric residence time as well as bioavailability and there by shows increased therapeutic efficacy. In vitro dissolution studies were performed for all formulations. Thus all formulations with HPMC K100 polymer were identified as ideal batch based on its results. Finally, it was concluded that HPMC can be successfully used in the formulation of pantoprazole sodium control release gastro retentive 
floating drug delivery system. The developed floating tablets of pantoprazole sodium may be used in clinic for prolonged drug release for at least $12^{\text {th }}$ thereby improving the bioavailability and patient compliance.

\section{REFERENCES}

1. Singh B.N, Kim K.N Floating Drug Delivery Systems: An Approach of Oral Controlled Drug Delivery Via Gastric Retention, J Controlled Release 2000; 63,235-59.

2. Patel MG, Patel RH, Patel M, Patel KJ. Floating drug delivery System: An innovative approach to prolong gastric retention. Available at WWW. Pharmainfo.net.aug2010.

3. Rouge N, Buri P, Doelker E. Drug absorption site in the gastrointestinal tract and dosage forms for site-specific delivery. Int J Pharm 1996; 136:117-39.

4. Mayavanshi AV, Gajjar SS, Floating drug delivery systems to increase gastric retention of drugs: A Review Research J. Pharm. And Tech. 1(4): Oct.-Dec.2008.

5. Prabhu P, Harish NM, Gulzar AM, Yadav B, Narayana CR, Satyanarayana D Et al. Formulation and in vitro evaluation of gastric oral floating tablets of glipizide. Indian $\mathbf{J}$ Pharm Educ Res 2008; 42(2): 174-83.

6. Chatterjee CC,. Human Physiology. Vol I, Medical Allied Agency; Kolkata; 2004; 435-8.

7. Garg S. and Sharma S. Gastro retentive Drug Delivery System, Business Briefing: Pharma Tech. 2003, 160-6.

8. Gattani YS, Floating drug delivery system: A Review, Int J Pharm and Bio Sciences 2010:V1(2) : 1-14.

9. Lachman L, Liebermann HA, Nicholas GI. Sustained released dosage forms, in; $2^{\text {nd }}$ Varghese publishing house, Mumbai, 1987; 439-40.

10. Levin M. Changing Tableting Machines in Scale-Up and Production: Ramifications for SUPAC Background Notes for FDA CDER DPqr Seminar April 3, 2000.

\section{AJPTR is}

- $\quad$ Peer-reviewed

- bimonthly

- Rapid publication

Submit your manuscript at: editor@ajptr.com

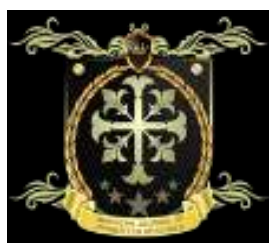

